\title{
MINERALIZACIÓN DE ETILENGLICOL POR FOTO-FENTON ASISTIDO CON FERRIOXALATO
}

\author{
Alba Nelly ARDILA ARIAS ${ }^{1 *}$, Erasmo ARRIOLA ${ }^{2}$, Juliana REYES CALLE ${ }^{1}$, \\ Eliana BERRIO MESA ${ }^{1}$ y Gustavo FUENTES ZURITA ${ }^{2}$
}

${ }^{1}$ Facultad de Ciencias Básicas Sociales y Humanas, Politécnico Colombiano Jaime Isaza Cadavid, Carrera 48 7-151 El Poblado, Medellín, Colombia, PBX: 3197900

${ }^{2}$ Departamento de Ingeniería de Procesos e Hidráulica, Universidad Autónoma Metropolitana, Campus Iztapalapa, San Rafael Atlixco 186, Colonia Vicentina, Iztapalapa, Ciudad de México, México, C.P. 09340

*Autor para correspondencia: anardila@elpoli.edu.co

(Recibido febrero 2015; aceptado noviembre 2015)

Palabras clave: agua residual de la industria de pinturas, ion ferroso, peróxido de hidrógeno, ion oxálico

\section{RESUMEN}

Se evaluó la efectividad del proceso foto-Fenton asistido con ferrioxalato para la mineralización de $1000 \mathrm{mg} / \mathrm{L}$ de etilenglicol presente en agua destilada y en agua residual proveniente de una planta de producción de pinturas. Se observó que la eficiencia de mineralización del etilenglicol es función de las concentraciones iniciales de ion ferroso, oxalato y peróxido de hidrógeno. Los mayores niveles de degradación del etilenglicol ( 90 y $85 \%$, para el agua destilada y el agua residual, respectivamente), se obtuvieron con $10 \mathrm{mg} / \mathrm{L}$ de $\mathrm{Fe}^{2+}, 150 \mathrm{mg} / \mathrm{L}$ de $\mathrm{C}_{2} \mathrm{O}_{4}^{2-}$ y $500 \mathrm{mg} / \mathrm{L}$ de $\mathrm{H}_{2} \mathrm{O}_{2}$, después de $3 \mathrm{~h}$ de irradiación artificial con lámparas UV a una longitud de onda máxima de $365 \mathrm{~nm}$. La descomposición del etilenglicol produjo cantidades traza $(<136 \mathrm{mg} / \mathrm{L})$ de ácido acético y ácido fórmico, por lo que la disminución en la demanda química de oxígeno debida a este contaminante fue prácticamente total, ya que no se detectó ningún otro producto intermediario. El hierro no actuó directamente de manera catalítica en el sistema.

Key words: wastewater from the paint industry, hydrogen peroxide, ferrous ion, oxalic ion

\begin{abstract}
The effectiveness of the ethylene glycol mineralization using ferrioxalate-induced photo-Fenton was evaluated. The efficiency of $1000 \mathrm{mg} / \mathrm{L}$ ethylene degradation was a function of the initial concentrations of ferrous ion, oxalate and hydrogen peroxide. The highest levels of degradation ( 90 and $85 \%$ for distilled water and wastewater from the paint industry, respectively) were obtained using $10 \mathrm{mg} / \mathrm{L}$ of Fe ${ }^{2+}, 150 \mathrm{mg} / \mathrm{L}$ of $\mathrm{C}_{2} \mathrm{O}_{4}^{2-}$ and $500 \mathrm{mg} / \mathrm{L}$ of $\mathrm{H}_{2} \mathrm{O}_{2}$ after 3 hours of UV artificial irradiation (wavelength $365 \mathrm{~nm}$ ). Ethylene glycol was converted to acetic acid and formic acid in trace amounts $(<136 \mathrm{mg} / \mathrm{L})$ resulting in a decrease of chemical oxygen demand due this contaminant. No other intermediate products were detected.
\end{abstract}




\section{INTRODUCCIÓN}

El etilenglicol es ampliamente utilizado en industrias dedicadas a la síntesis de productos farmacéuticos, cosméticos, polímeros, coadyuvantes agrícolas, plásticos, pinturas y recubrimientos, además, es usado en grandes cantidades como anticongelante y en los aeropuertos como agente de deshielo (McGinnis et al. 2000, McGinnis et al. 2001, Araña et al. 2012). Dados los usos y aplicaciones de este disolvente, no es difícil deducir que grandes cantidades de él formarán parte de las descargas de los efluentes acuosos y orgánicos producidos por dichas empresas.

El tratamiento de aguas residuales industriales contaminadas con etilenglicol es importante, ya que a pesar de su baja toxicidad en comparación con otros contaminantes acuíferos, la liberación de grandes cantidades de aguas residuales industriales conteniendo dicho compuesto puede afectar la vida animal y vegetal de los diferentes ecosistemas acuáticos. Esto se debe a su alta demanda química de oxígeno (DQO), lo cual promueve anoxia y eutrofización (Araña et al. 2012), además de que su contribución en forma de carbono orgánico total (COT) a las aguas residuales puede provocar un incremento en los costos de los sistemas de tratamiento de las aguas residuales municipales.

Por otra parte, en la Norma Técnica Colombiana (NTC) 6018 de 2013, cuyo objetivo principal es identificar y proponer diferentes medidas que permitan controlar y reducir los impactos ambientales generados por las materias primas usadas en la fabricación de pinturas y materiales de recubrimiento, se establece restringir y minimizar el contenido de etilenglicol. Sin embargo, de acuerdo con datos reportados por el Ministerio de Comercio Exterior de la República de Colombia, este disolvente orgánico se importa en grandes cantidades a Colombia y se comercializa principalmente en la industria de pinturas y materiales de recubrimiento (SIEX 2015). Además, de acuerdo con la búsqueda realizada en la literatura, a la fecha no existe una normatividad nacional o internacional clara con respecto al manejo de este disolvente, ni mucho menos se tienen mecanismos específicos para la gestión de aguas residuales contaminadas con el mismo.

Se han estudiado sistemas de reciclaje, biodegradación y de oxidación avanzada para el tratamiento de residuos que contienen etilenglicol, obteniéndose los mejores porcentajes de degradación con los tratamientos Fenton y foto-Fenton (McGinnis et al. 2000, McGinnis et al. 2001, Araña et al. 2012).
Estas dos últimas tecnologías de oxidación avanzada son una alternativa viable y eficaz para la disminución del impacto ambiental generado por las aguas residuales contaminadas con este disolvente (Aguirre 2010). Sin embargo, a la fecha las investigaciones publicadas y enfocadas a la degradación de dicho contaminante usando estos métodos son muy escasas (McGinnis et al. 2000, McGinnis et al. 2001, Araña et al. 2012). Además, en dichos sistemas la degradación del etilenglicol se llevó a cabo usando soluciones ideales del mismo, lo que conlleva a que se ignoren los efectos de otros contaminantes que pueden estar presentes en las aguas residuales reales.

Una versión mejorada de los sistemas Fenton $\mathrm{y}$ foto-Fenton consiste en el sistema foto-Fenton asistido con ferrioxalato $\left(\mathrm{UV} / \mathrm{H}_{2} \mathrm{O}_{2} / \mathrm{Fe}^{2+} / \mathrm{C}_{2} \mathrm{O}_{4}^{2-}\right.$ ), cuya eficiencia en la degradación de diferentes compuestos orgánicos tóxicos ha sido demostrada muy recientemente (Prato et al. 2009, Salem et al. 2009, Monteagudo et al. 2010). La irradiación del ferrioxalato en una solución ácida genera dióxido de carbono y iones $\mathrm{Fe}^{2+}$ libres o formando complejos con el oxalato, los cuales en combinación con el $\mathrm{H}_{2} \mathrm{O}_{2}$ proveen una fuente constante del reactivo Fenton (Ecuaciones 1-5). La gran eficiencia de este proceso en comparación con los otros procesos oxidativos es atribuida al hecho de que el ferrioxalato absorbe en un amplio rango de longitudes de onda (200-450 nm), es decir, utiliza más eficientemente la radiación UV-Vis (Selvam et al. 2005). En algunos casos, esta característica favorece su aplicación en el tratamiento de aguas residuales usando la energía solar como fuerte de irradiación.

Además, el rendimiento cuántico de formación del $\mathrm{Fe}^{2+}$ es 1.0-1.2 en dicho intervalo de irradiación, mientras que en el proceso foto-Fenton el rendimiento cuántico para la formación del $\mathrm{Fe}^{2+}$ disminuye de 0.14 a 0.017 al aumentar la longitud de onda de 313 a $360 \mathrm{~nm}$. Por otra parte, el sistema $\mathrm{UV} / \mathrm{H}_{2} \mathrm{O}_{2} / \mathrm{Fe}^{2+}$ / $\mathrm{C}_{2} \mathrm{O}_{4}^{2-}$ tiene la ventaja de requerir menor tiempo de irradiación, tiene mayor capacidad de degradación de contaminantes tóxicos y permite operar en un intervalo más amplio de $\mathrm{pH}$. De hecho, se puede operar prácticamente a pH 7 debido a la formación del complejo hierro-oxalato. Adicionalmente, estos complejos tienen mayor fotorreactividad que los complejos formados en foto-Fenton como el $\mathrm{Fe}(\mathrm{OH})^{2+}$ (Machulek et al. 2012, Shanthi et al. 2013).

En este trabajo se reportan los resultados obtenidos en la degradación de etilenglicol por fotoFenton asistido con ferrioxalato. En el estudio se establecen las condiciones óptimas del tratamiento 
estudiando el efecto de varios factores: concentración inicial de $\mathrm{Fe}^{2+}, \mathrm{C}_{2} \mathrm{O}_{4}^{2-}$ y $\mathrm{H}_{2} \mathrm{O}_{2}$. Además, una vez establecidas las mejores condiciones de reacción, éstas se aplicaron en la degradación del etilenglicol presente en el efluente de una planta de producción de pinturas.

\section{MATERIALES Y MÉTODOS}

Experimentos testigo. Antes de optimizar los parámetros para los ensayos foto-Fenton asistidos con ferrioxalato, se realizaron cuatro tipos de pruebas como experimentos testigo usando una solución de $1000 \mathrm{mg} / \mathrm{L}$ de etilenglicol.

Experimento a) Sistema etilenglicol/UV: la solución se sometió a fotólisis $\left(\lambda_{\max }=365 \mathrm{~nm}\right)$ en ausencia de hierro, peróxido de hidrógeno y ácido oxálico con el fin de determinar la acción degradativa de la radiación UV y el efecto térmico producido por las lámparas.

Experimento b) Sistema etilenglicol/ $\mathrm{UV} / \mathrm{H}_{2} \mathrm{O}_{2}$ : se estudió la acción oxidativa usando $500 \mathrm{mg} / \mathrm{L}$ de peróxido de hidrógeno y radiación $U V\left(\lambda_{\max }=365 \mathrm{~nm}\right)$.

Experimento c) Sistema etilenglicol $/ \mathrm{Fe}^{2+} /$ $\mathrm{H}_{2} \mathrm{O}_{2}$ : se evaluó la efectividad del reactivo Fenton tradicional usando $10 \mathrm{mg} / \mathrm{L}$ de $\mathrm{Fe}^{2+}$ y $500 \mathrm{mg} / \mathrm{L}$ de $\mathrm{H}_{2} \mathrm{O}_{2}$.

Experimento d) Sistema etilenglicol/ $\mathrm{UV} / \mathrm{Fe}^{2+} /$ $\mathrm{H}_{2} \mathrm{O}_{2}$ : se investigó la eficiencia del tratamiento fotoFenton sometiendo a radiación UV $\left(\lambda_{\max }=365 \mathrm{~nm}\right)$ usando $10 \mathrm{mg} / \mathrm{L}$ de $\mathrm{Fe}^{2+}$ y $500 \mathrm{mg} / \mathrm{L}$ de $\mathrm{H}_{2} \mathrm{O}_{2}$.

Experimento e) Etilenglicol/ $\mathrm{UV} / \mathrm{Fe}^{2+} / \mathrm{C}_{2} \mathrm{O}_{4}^{2-}$ : finalmente se determinó la efectividad de los complejos de ferrioxalato en la degradación del etilenglicol en ausencia de peróxido de hidrógeno, $10 \mathrm{mg} / \mathrm{L}$ de $\mathrm{Fe}^{2+}$ y $150 \mathrm{mg} / \mathrm{L}$ de $\mathrm{C}_{2} \mathrm{O}_{4}^{2-}$ bajo radiación $\mathrm{UV}\left(\lambda_{\max }\right.$ $=365 \mathrm{~nm}$ ).

Todas las pruebas correspondientes a los experimentos testigo se llevaron a cabo con el $\mathrm{pH}$ natural de la solución de etilenglicol, el cual correspondió a un valor $\sim 4.0-4.5$. Después de $3 \mathrm{~h}$ de reacción no se observaron actividades fotocatalíticas apreciables en las pruebas que permitieran obtener valores aceptables para realizar algunas comparaciones entre dichos tratamientos. La eficiencia de este tipo de tratamientos oxidativos depende fuertemente de las condiciones de reacción y posiblemente pueden requerir tiempos prolongados para obtener porcentajes de degradación significativos. Por lo tanto, dichos sistemas se evaluaron a un tiempo total de $24 \mathrm{~h}$ para tener un mejor punto de comparación.

\section{Determinación de las condiciones óptimas para la degradación del etilenglicol presente en una solución ideal}

Las pruebas se realizaron a temperatura ambiente en reactores cilíndricos de borosilicato de $50 \mathrm{~mL}$ (diámetro $6.5 \mathrm{~cm}$, altura $4.5 \mathrm{~cm}$ ) con $20 \mathrm{~mL}$ de una solución de etilenglicol con una concentración inicial igual a $1000 \mathrm{mg} / \mathrm{L}$ (387 mg/L de carbono orgánico total). Las pruebas foto-Fenton se hicieron mediante irradiación ultravioleta (UV) artificial suministrada por dos lámparas fluorescentes de luz negra marca Tecnolite con una longitud de onda máxima de $~ 365$ $\mathrm{nm}$ y $15 \mathrm{~W}$ de potencia, ubicadas en una cabina de fotocatálisis de dimensiones interiores o de área de trabajo efectiva de $40 \mathrm{~cm}$ ancho por $25 \mathrm{~cm}$ largo y $19 \mathrm{~cm}$ altura. (Fig. 1). Todas las muestras se mantuvieron bajo agitación magnética a $260 \mathrm{rpm}$ durante un tiempo de reacción total de $3 \mathrm{~h}$. Una vez finalizados los ensayos, las muestras fueron filtradas a través de membranas de celulosa con diámetro de poro de $0.45 \mu \mathrm{m}$.

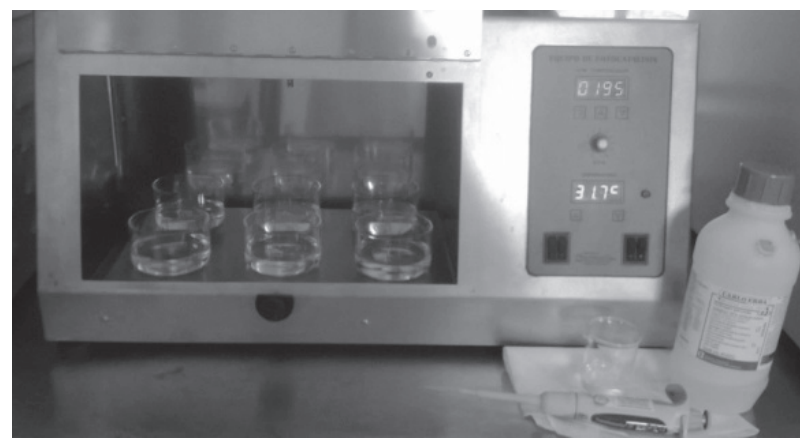

Fig. 1. Cabina de fotocatálisis y reactores utilizados durante el proceso

Diseño experimental. Se realizaron diferentes pruebas con el objetivo de establecer las condiciones óptimas del tratamiento foto-Fenton asistido con ferrioxalato sobre la eficiencia de degradación del etilenglicol, para lo cual se aplicó un diseño experimental factorial basado en la incidencia de varios factores (concentraciones iniciales de $\mathrm{Fe}^{2+}, \mathrm{C}_{2} \mathrm{O}_{4}^{2-}$ y $\mathrm{H}_{2} \mathrm{O}_{2}$ ). Los factores con sus correspondientes niveles se presentan en el cuadro I.

El diseño experimental aplicado se basó en un modelo factorial asimétrico, debido a que el número de factores que se analizaron es diferente al número de niveles. Esto se realizó con el fin de reducir el tamaño del diseño factorial, ya que no es necesario realizar todos los experimentos por completo para obtener la información deseada. Todos los ensayos fotocatalíticos y mediciones se realizaron por duplicado. 
CUADRO I. FACTORES Y NIVELES DEL DISEÑO EXPERIMENTAL

\begin{tabular}{llrrr}
\hline \multirow{2}{*}{ Factores } & \multicolumn{3}{c}{ Niveles $(\mathrm{mg} / \mathrm{L})$} \\
\cline { 3 - 5 } & & \multicolumn{1}{c}{$i$} & \multicolumn{1}{c}{$J$} & $k$ \\
\hline$\alpha$ & Concentración inicial de $\mathrm{Fe}^{2+}$ & 10 & 50 & 100 \\
$\beta$ & Concentración inicial de $\mathrm{C}_{2} \mathrm{O}_{4}^{2-}$ & 50 & 150 & \\
$\gamma$ & Concentración inicial de $\mathrm{H}_{2} \mathrm{O}_{2}$ & 250 & 500 & \\
\hline
\end{tabular}

Los espectros UV de las soluciones fueron obtenidos con un espectrofotómetro UV-Vis-NIR Cary/5E marca Varian. La cuantificación del etilenglicol, ácido acético y ácido fórmico se realizó utilizando curvas de calibración con acetonitrilo como estándar interno. Las muestras se analizaron en un cromatógrafo de gases Agilent 6890 equipado con un detector de ionización de llama (FID) y una columna capilar HP-Innowax. La concentración de $\mathrm{H}_{2} \mathrm{O}_{2}$ al final de las reacciones se determinó por titulación usando una solución de $\mathrm{KMnO}_{4} 0.01 \mathrm{M}$.

La temperatura y la intensidad de la luz incidente del sistema no fueron controladas, sin embargo, éstas se midieron durante la reacción y sus valores promedio fueron $35^{\circ} \mathrm{C}$ y $615 \mathrm{~kJ} / \mathrm{m}^{2}$. Esta última se determinó usando un radiómetro marca Eppley modelo PSP. El cálculo de las especies presentes en equilibrio en la solución en función del $\mathrm{pH}$ del medio (diagrama de especiación), se realizó con el programa Medusa Hydra.

\section{Degradación del etilenglicol presente en un efluen- te real bajo las mejores condiciones}

Las aguas residuales de la industria de pinturas, además de poseer altas concentraciones de etilenglicol, contienen cantidades elevadas de materia orgánica como fenoles, grasas y aceites junto con metales pesados. Estos contaminantes pueden afectar el proceso de degradación del etilenglicol y probablemente habría que optimizar nuevamente las dosificaciones de cada agente químico. Por lo tanto, para evaluar el desempeño de las condiciones óptimas, se utilizó un efluente real de una planta de producción de pinturas ubicada en el municipio la Estrella, Antioquia.

\section{Jornada de aforo y muestreo}

Para recolectar el efluente se realizó una jornada de aforo y un plan de muestreo, esto con el fin de obtener una muestra compuesta representativa. El muestreo se llevó a cabo durante $30 \mathrm{~min}$, tiempo empleado para el lavado con agua potable de tanques y tolvas que se encuentran contaminadas con pinturas fabricadas a base de solventes orgánicos tales como fenol, xileno, acetato de etilo y etilenglicol. Durante este periodo se tomaron ocho muestras simples cada 4 min, cada una con un volumen igual a $120 \mathrm{~mL}$, a las cuales se les midió inmediatamente propiedades fisicas y químicas como caudal, temperatura, $\mathrm{pH}$, conductividad, turbidez, color, sólidos disueltos y sólidos totales. A partir de estas muestras simples se formó una muestra compuesta y se realizó la medición de los mismos parámetros y adicionalmente sólidos sedimentables, oxígeno disuelto, demanda química de oxígeno, demanda bioquímica de oxígeno y carbono orgánico total. Cada muestra se almacenó en recipientes de plástico de alta densidad a $4{ }^{\circ} \mathrm{C}$ y se adicionaron unas gotas de ácido clorhídrico grado reactivo para evitar el crecimiento de microorganismos. Finalmente, se conservó y vigiló todas las muestras de manera adecuada para garantizar la integridad de la cadena de custodia de las mismas.

El tratamiento del agua residual se realizó utilizando el mismo sistema de degradación bajo las mejores condiciones de reacción encontradas: $\left[\mathrm{Fe}^{3+}\right]$ $=10 \mathrm{mg} / \mathrm{L},\left[\mathrm{C}_{2} \mathrm{O}_{4}^{2-}\right]=150 \mathrm{mg} / \mathrm{Ly}\left[\mathrm{H}_{2} \mathrm{O}_{2}\right]=500 \mathrm{mg} / \mathrm{L}$. La cuantificación de etilenglicol se realizó por cromatografía de gases. Además, se determinó el porcentaje de remoción de DQO, $\mathrm{DBO}_{5}$ y COT. El seguimiento de estos parámetros se realizó en un laboratorio acreditado siguiendo las normas de la APHA, AWWA y WEF para el análisis de aguas superficiales y aguas residuales.

\section{RESULTADOS Y DISCUSIÓN}

El espectro de absorción del etilenglicol en el intervalo UV tiene un valor máximo de absorbancia a $185 \mathrm{~nm}$ (Fig. 2). En el espectro se observa la capacidad del etilenglicol para absorber radiación

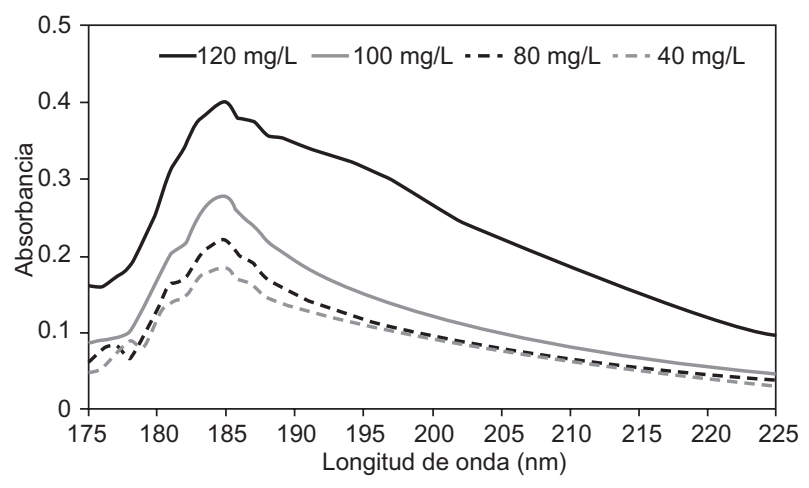

Fig. 2. Espectro de absorción UV del etilenglicol a diferentes concentraciones 
de la longitud de onda incidente suministrada por las lámparas artificiales usadas en la presente investigación, cuyo valor máximo de longitud de onda es $\sim 365 \mathrm{~nm}$.

\section{Experimentos testigo}

Experimento a) Es conocido que la radiación UV tiene la capacidad de romper enlaces químicos en moléculas complejas para su mineralización (García et al. 1997) mediante la absorción de fotones que tengan una energía mayor que las de los enlaces del compuesto que se desea destruir, generando radicales libres que participan en una serie de reacciones hasta obtener productos e intermediarios de la misma reacción (ecuaciones 1 y 2). Sin embargo, este mecanismo tiene baja eficacia en la degradación y mineralización de contaminantes orgánicos, requiriendo tiempos de reacción largos (Andreozzi et al. 1999, Saritha et al. 2007). Esto se comprueba con los resultados del cuadro II, los cuales muestran que al utilizar sólo radiación UV el porcentaje de degradación de etilenglicol es despreciable (2.1\%).

CUADRO II. RESULTADOS DE EXPERIMENTOS TESTIGO

\begin{tabular}{lc}
\hline $\begin{array}{l}\text { Experimentos testigo } \\
(24 \mathrm{~h})\end{array}$ & $\begin{array}{c}\text { Degradación etilenglicol } \\
(\%)\end{array}$ \\
\hline Etilenglicol/UV & 2.1 \\
Etilenglicol/ $/ \mathrm{UV} / \mathrm{H}_{2} \mathrm{O}_{2}$ & 6.3 \\
Etilenglicol $/ \mathrm{Fe}^{2+} / \mathrm{H}_{2} \mathrm{O}_{2}$ & 11.7 \\
Etilenglicol $/ \mathrm{UV} / \mathrm{Fe}^{2+} / \mathrm{H}_{2} \mathrm{O}_{2}$ & 25.8 \\
Etilenglicol/UV/ $/ \mathrm{Fe}^{2+} / \mathrm{C}_{2} \mathrm{O}_{4}^{2-}$ & 15.2 \\
\hline
\end{tabular}

Compuesto $+h v \rightarrow$ Intermediarios

Intermediarios $+h v \rightarrow \mathrm{CO}_{2}+\mathrm{H}_{2} \mathrm{O}+\mathrm{Cl}^{-}$

Experimento b) La generación de radicales hidroxilo $\left(\mathrm{OH}^{*}\right)$ a partir de la fotólisis del $\mathrm{H}_{2} \mathrm{O}_{2}$ y subsecuentes reacciones de propagación (Saritha et al. 2007) puede favorecer la descomposición de moléculas orgánicas (Ec. 3). Sin embargo, dicho sistema no favoreció la degradación del etilenglicol de manera significativa $(6.3 \%)$, posiblemente porque durante todo el tiempo de reacción se trabajó a una longitud de onda próxima al visible $(\sim 365$ $\mathrm{nm}$ ), región en la cual no ocurre en gran proporción fotólisis del $\mathrm{H}_{2} \mathrm{O}_{2}$. El mayor rendimiento cuántico de este compuesto se da entre longitudes de onda de 220-250 nm, donde hay rompimiento del enlace O-O, que genera radicales hidroxilo (Aguirre 2010). Esto se comprobó al cuantificar el $\mathrm{H}_{2} \mathrm{O}_{2}$ después de la irradiación, el cual no cambió significativamente (490 mg/L).

$\mathrm{H}_{2} \mathrm{O}_{2}+h v \rightarrow 2 \mathrm{OH}^{\cdot}$

Experimento c) El proceso Fenton tradicional es comúnmente utilizado para la destrucción de sustancias orgánicas tóxicas debido a la generación de radicales hidroxilo $\left(\mathrm{OH}^{\circ}\right)$ por la adición de $\mathrm{H}_{2} \mathrm{O}_{2}$ a sales de $\mathrm{Fe}^{2+}$ mediante una serie de reacciones complejas en cadena (ecuaciones 4 a 6) (Saritha et al. 2007). Sin embargo, sólo se degradó un $11.7 \%$ de etilenglicol después de $24 \mathrm{~h}$ de reacción. La necesidad de controlar el $\mathrm{pH}$ de la solución es una de las mayores desventajas del proceso Fenton, el cual debe permanecer en un intervalo de 2.1-3.4 para garantizar principalmente la formación de radicales hidroxilo $\left(\mathrm{OH}^{*}\right)$ (McGinnis et al. 2000). No obstante, en el presente sistema no se controló dicho parámetro, lo cual probablemente afectó la eficiencia del proceso.

$$
\begin{aligned}
& \mathrm{Fe}^{2+}+\mathrm{H}_{2} \mathrm{O}_{2} \rightarrow \mathrm{Fe}^{3+}+\mathrm{OH}^{-}+\mathrm{OH}^{\bullet} \\
& \mathrm{Fe}^{3+}+\mathrm{H}_{2} \mathrm{O}_{2} \rightleftarrows \mathrm{H}^{+}+\mathrm{FeOOH}^{2+} \\
& \mathrm{FeOOH}^{2+} \rightarrow \mathrm{Fe}^{2+}+\mathrm{OH}_{2}^{\cdot}
\end{aligned}
$$

Experimento d) Una extensión del sistema Fenton tradicional se basa en el uso de dicho reactivo en combinación con irradiación a longitudes de onda mayores de $300 \mathrm{~nm}$ (Saritha et al. 2007), lo cual permite la regeneración de iones $\mathrm{Fe}^{2+}$ mediante la fotorreducción de complejos de $\mathrm{Fe}^{3+}$ (Ghiselli et al. 2004, Kusic et al. 2006), junto con la producción de radicales hidroxilo (ecuaciones 4 y 7). Éstos aumentan la degradación de contaminantes orgánicos con respecto al tratamiento Fenton simple. Sin embargo, a pesar de ser un método eficiente, el $\mathrm{H}_{2} \mathrm{O}_{2}$ debe ser agregado constantemente y se debe controlar el pH del sistema en un valor entre 3 y 4 para evitar la precipitación del $\mathrm{Fe}^{2+}$ como hidróxido o sales (Nogueira et al. 2005, Giroto et al 2010). Como se muestra en el cuadro II, el porcentaje de degradación de etilenglicol fue de sólo $25.8 \%$ después de 24 h de reacción. La cuantificación del $\mathrm{H}_{2} \mathrm{O}_{2}$ realizada después de este tiempo demostró que se descompuso completamente durante el proceso, lo cual pudo haber limitado la degradación del etilenglicol. Además, como se mencionó anteriormente, el $\mathrm{pH}$ de la solución controla la velocidad de producción de los radicales hidroxilo y la naturaleza de las especies de hierro presentes en la solución, sin embargo, este parámetro tampoco se controló durante el tratamiento. 
$\mathrm{Fe}(\mathrm{OH})^{2+}+h v \rightarrow \mathrm{Fe}^{2+}+\mathrm{OH}^{\cdot}$

Experimento e) El porcentaje de degradación de etilenglicol fue muy bajo con el sistema $\mathrm{Fe}^{2+}$ / $\mathrm{C}_{2} \mathrm{O}_{4}^{2-} / \mathrm{UV}(15.2 \%)$ bajo las condiciones de reacción aplicadas en el presente trabajo, esto se debe probablemente a la ausencia del agente oxidante $\left(\mathrm{H}_{2} \mathrm{O}_{2}\right)$. Diferentes investigaciones han demostrado que los complejos de ferrioxalato aceleran la producción de radicales hidroxilo en presencia de $\mathrm{H}_{2} \mathrm{O}_{2}$ (Tran et al. 2013). Además, los mismos complejos tienen la capacidad de reaccionar también con el oxígeno disuelto en la solución para formar radicales superóxido y posteriormente $\mathrm{H}_{2} \mathrm{O}_{2}$, los cuales a su vez incrementan la velocidad de generación de radicales hidroxilo (Vedrenne et al. 2012). Por lo tanto, este proceso es poco eficiente cuando no se adiciona $\mathrm{H}_{2} \mathrm{O}_{2}$. Esto se debe a que la reacción Fenton está limitada por la formación de $\mathrm{H}_{2} \mathrm{O}_{2}$, y aunque en las etapas iniciales de irradiación el $\mathrm{H}_{2} \mathrm{O}_{2}$ se forma de acuerdo con las ecuaciones 8-10, éste es insuficiente para que la reacción Fenton ocurra, incluso aunque el $\mathrm{Fe}^{2+}$ sea regenerado continuamente por la fotólisis del ferrioxalato en el sistema $\mathrm{UV} / \mathrm{Fe}^{2+} / \mathrm{C}_{2} \mathrm{O}_{4}^{2-}$ (Kusic et al. 2006).

$\mathrm{Fe}^{2+}+\mathrm{O}_{2}^{--} \rightarrow \mathrm{Fe}^{3+}+\mathrm{H}_{2} \mathrm{O}_{2}$

$2 \mathrm{HO}_{2}^{*} \rightarrow \mathrm{H}_{2} \mathrm{O}_{2}+\mathrm{O}_{2}$

$\mathrm{HO}_{2}^{\cdot}+\mathrm{Fe}^{2+}+\mathrm{H}^{+} \rightarrow \mathrm{Fe}^{3+}+\mathrm{H}_{2} \mathrm{O}_{2}$

En conclusión, los resultados obtenidos en todos los experimentos testigo mostraron bajos porcentajes de degradación del etilenglicol aún en tiempos de reacción largos.

Degradación del etilenglicol presente en una solución ideal usando el sistema foto-Fenton asistido con ferrioxalato

Los complejos de ferrioxalato pueden obtenerse de la combinación de reactivos como el $\mathrm{Fe}^{2+}$ y el oxalato (ecuaciones 11 a 12). Además, si se agrega $\mathrm{H}_{2} \mathrm{O}_{2}$ al proceso, la reducción fotoquímica del complejo de $\mathrm{Fe}^{3+}$ se acopla a la reacción Fenton generándose $\mathrm{Fe}^{2+}$-oxalato que reacciona rápidamente con $\mathrm{H}_{2} \mathrm{O}_{2}$ generando radicales $\mathrm{OH}^{*}$ (ecuaciones 4 y 13 a 17).

$$
\begin{aligned}
& \mathrm{Fe}^{2+}+\mathrm{C}_{2} \mathrm{O}_{4}^{2-} \rightleftarrows \mathrm{Fe}\left(\mathrm{C}_{2} \mathrm{O}_{4}\right) \\
& \mathrm{Fe}\left(\mathrm{C}_{2} \mathrm{O}_{4}\right)+\mathrm{H}_{2} \mathrm{O}_{2} \rightarrow \mathrm{Fe}\left(\mathrm{C}_{2} \mathrm{O}_{4}\right)^{+}+\mathrm{HO}^{\bullet}+\mathrm{HO}^{-} \\
& \mathrm{HO}_{2}^{+}+\mathrm{HO}_{2}^{*} \rightarrow \mathrm{H}_{2} \mathrm{O}_{2}+\mathrm{O}_{2}
\end{aligned}
$$

$$
\begin{aligned}
& {\left[\mathrm{Fe}\left(\mathrm{C}_{2} \mathrm{O}_{4}\right)_{3}\right]^{3-}+h v \rightarrow \mathrm{Fe}^{2+}+2 \mathrm{C}_{2} \mathrm{O}_{4}^{2-}+\mathrm{C}_{2} \mathrm{O}_{4}^{--}} \\
& \mathrm{C}_{2} \mathrm{O}_{4}^{--}+\left[\mathrm{Fe}\left(\mathrm{C}_{2} \mathrm{O}_{4}\right)_{3}\right]^{3-} \rightarrow \mathrm{Fe}^{2+}+3 \mathrm{C}_{2} \mathrm{O}_{4}^{2-}+2 \mathrm{CO}_{2} \\
& \mathrm{C}_{2} \mathrm{O}_{4}^{--}+\mathrm{O}_{2} \rightarrow 2 \mathrm{CO}_{2}+\mathrm{O}_{2}^{--} \\
& \mathrm{O}_{2}^{-}+\mathrm{H}^{+} \rightarrow \mathrm{HO}_{2}^{-}
\end{aligned}
$$

Otra característica de este sistema es la capacidad de generar $\mathrm{H}_{2} \mathrm{O}_{2}$ in situ en medio acuoso como se muestran en las ecuaciones 18 a 21 (Aguirre 2010, Monteagudo et al. 2010). La ecuación 14 tiene un elevado rendimiento cuántico $(\phi=1.0$ 1.2), casi independiente de la longitud de onda, por eso el uso de mezclas $\mathrm{H}_{2} \mathrm{O}_{2}$-ferrioxalato bajo irradiación es muy eficiente para la degradación de compuestos orgánicos, sin embargo, la eficiencia del proceso depende fuertemente de las condiciones de reacción.

$$
\begin{aligned}
& \mathrm{Fe}^{2+}+\mathrm{O}_{2}^{--}+2 \mathrm{H}^{+} \rightarrow \mathrm{Fe}^{3+}+\mathrm{H}_{2} \mathrm{O}_{2} \\
& \mathrm{O}_{2}^{--}+\mathrm{O}_{2} \rightarrow \mathrm{CO}_{2}+\mathrm{O}_{2}^{-} \\
& \mathrm{C}_{2} \mathrm{O}_{4}^{--} \longrightarrow \mathrm{CO}_{2}^{--}+\mathrm{CO}_{2} \\
& \mathrm{HO}_{2} \rightleftarrows \mathrm{O}_{2}^{--}+\mathrm{H}^{+}
\end{aligned}
$$

\section{Análisis estadístico}

Se utilizó un diseño factorial de tres factores y se realizó el respectivo análisis de varianza con el paquete estadístico StatGraphics 15.1 y la ecuación 22.

$y_{i j k}=\mu+\alpha_{i}+\beta_{j}+\gamma_{k}+(\alpha \beta)_{i j}+(\beta \gamma)_{j k}+(\alpha \gamma)_{i k}+\varepsilon_{i j k}$

Donde:

$y_{i j k} \quad$ Es la medida del porcentaje de degradación de etilenglicol, observado en los respectivos niveles de los tres factores bajo estudio.

$\mu \quad$ Es el efecto global promedio.

$\alpha_{i} \quad$ Es el efecto del i-ésimo nivel del factor concentración inicial de $\mathrm{Fe}^{2+}$.

$i \quad 10,50$ y $100 \mathrm{mg} / \mathrm{L}$.

$\beta_{j} \quad$ Es el efecto del j-ésimo nivel del factor concentración inicial del $\mathrm{C}_{2} \mathrm{O}_{4}^{2-}$.

j 50 y $150 \mathrm{mg} / \mathrm{L}$.

$\gamma_{k} \quad$ Es el efecto del k-ésimo nivel del factor concentración inicial de $\mathrm{H}_{2} \mathrm{O}_{2}$.

$k \quad 250$ y $500 \mathrm{mg} / \mathrm{L}$.

$(\alpha \beta)_{i j}$ Es el efecto de la interacción entre los niveles de los factores concentración inicial de $\mathrm{Fe}^{2+}$ $\mathrm{y}_{2} \mathrm{O}_{4}^{2-}$. 
$(\beta \gamma)_{j k}$ Es el efecto de la interacción entre los niveles de los factores concentración inicial de $\mathrm{C}_{2} \mathrm{O}_{4}^{2-}$ $\mathrm{y}_{2} \mathrm{O}_{2}$.

$(\alpha \gamma)_{i k}$ Es el efecto de la interacción entre los niveles de los factores concentración inicial de $\mathrm{Fe}^{2+}$ $\mathrm{y}_{2} \mathrm{O}_{2}$.

$\varepsilon_{i j k} \quad$ Es el efecto atribuido al error aleatorio, que en este diseño es representado por la interacción entre los tres factores, que se supone no debe ser significativa y con esto permite la estimación de las varianzas.

Se utilizó un valor de nivel de significancia igual a 0.05 , y se estableció como hipótesis nula $\left(\mathrm{H}_{0}\right)$ que las medias del porcentaje de degradación de etilenglicol son iguales frente a los diferentes factores estudiados. En el cuadro III, se muestran los resultados aplicados en el modelo de análisis de varianza. Los resultados indican que los parámetros analizados influyen significativamente en la degradación fotocatalítica del etilenglicol con un nivel de confianza del $95 \%$, es decir, un error máximo permisible del $5 \%$. Todos aquellos factores o interacciones que obtuvieron un valor $\mathrm{P}<$ 0.05 se pueden considerar como los más relevantes en el proceso de degradación del etilenglicol. Por ejemplo, el cuadrado medio del factor A (concentración inicial del ion $\mathrm{Fe}^{2+}$ ) es mayor que el cuadrado medio del error (F), es decir, $1811.17>114.39$, esto indica que no es posible que los porcentajes medios de degradación de etilenglicol sean iguales a diferentes concentraciones iniciales del $\mathrm{Fe}^{2+}$.

Por otro lado, el cociente F de la interacción concentración inicial de $\mathrm{Fe}^{2+}$ concentración inicial de $\mathrm{C}_{2} \mathrm{O}_{4}^{2-}$ (222.167) tiene un valor $\mathrm{P}$ de 0.0007 , lo que indica que existe una fuerte interacción entre estos dos factores. En otras palabras, al analizar los datos obtenidos, se puede rechazar la $\mathrm{H}_{0}$ y concluir que el porcentaje medio de degradación de etilenglicol de los tratamientos difiere, es decir, hay una interacción significativa entre el valor de la concentración inicial de $\mathrm{Fe}^{2+}, \mathrm{C}_{2} \mathrm{O}_{4}^{2-}$ y $\mathrm{H}_{2} \mathrm{O}_{2}$, para cada parámetro. Igualmente, se puede afirmar que los efectos cruzados principales corresponden a la interacción $\mathrm{Fe}^{2+}$ / $\mathrm{C}_{2} \mathrm{O}_{4}^{2-}$ y $\mathrm{Fe}^{2+} / \mathrm{H}_{2} \mathrm{O}_{2}$.

\section{Determinación de las condiciones óptimas}

Como se muestra en la figura 3 , el porcentaje de degradación del etilenglicol incrementa con la disminución en la relación $\mathrm{Fe}^{2+} / \mathrm{C}_{2} \mathrm{O}_{4}^{2-}$, sin embargo, el efecto no es tan notable a relaciones menores de 0.45 . Esto significa que la degradación disminuye con el incremento de la concentración inicial de $\mathrm{Fe}^{2+}$ y aumenta con el aumento en la concentración inicial de $\mathrm{C}_{2} \mathrm{O}_{4}^{2-}$. A su vez, esto indica que en el intervalo de concentraciones estudiado el ion $\mathrm{Fe}^{2+}$ se encuentra como reactivo en exceso y el ion $\mathrm{C}_{2} \mathrm{O}_{4}^{2-}$ como reactivo limitante. Esto sugiere que la fotodegradación catalítica aumentó al favorecer la formación de los complejos de ferrioxalato debido al incremento en la concentración del ion $\mathrm{C}_{2} \mathrm{O}_{4}^{2-}$.

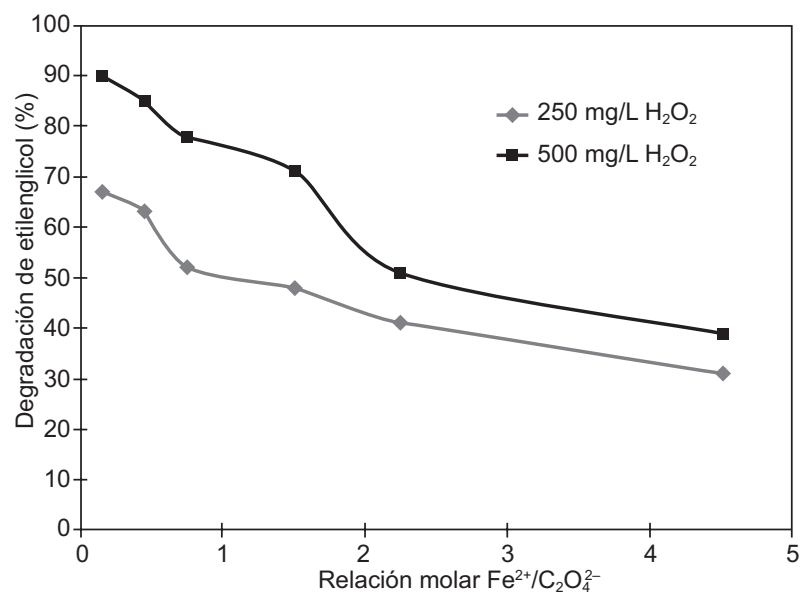

Fig. 3. Porcentaje de degradación de etilenglicol después de 3 $\mathrm{h}$ de irradiación en función de la relación molar $\mathrm{Fe}^{2+}$ / $\mathrm{C}_{2} \mathrm{O}_{4}^{2-}$ a diferentes concentraciones iniciales de $\mathrm{H}_{2} \mathrm{O}_{2}$

CUADRO III. RESULTADOS DEL ANÁLISIS DE VARIANZA

\begin{tabular}{lccccc}
\hline \multicolumn{5}{c}{ Degradación de etilenglicol (\%) } \\
\hline Fuente de variación & GL & Suma de cuadrados & Cuadrado medio & Relación F & Valor de P \\
\hline Factor $\alpha:\left[\mathrm{Fe}^{2+}\right]$ & 2 & 3622.33 & 1811.17 & 114.39 & 0.0000 \\
Factor $\beta:\left[\mathrm{C}_{2} \mathrm{O}_{4}^{2-}\right]$ & 1 & 1568.17 & 1568.17 & 99.04 & 0.0000 \\
Factor $\gamma:\left[\mathrm{H}_{2} \mathrm{O}_{2}\right]$ & 1 & 1441.5 & 1441.5 & 91.04 & 0.0000 \\
$\alpha \beta$ & 2 & 444.333 & 222.167 & 14.03 & 0.0007 \\
$\alpha \gamma$ & 2 & 211.0 & 105.5 & 6.66 & 0.0113 \\
$\beta \gamma$ & 1 & 433.5 & 433.5 & 27.38 & 0.0002 \\
$\gamma \beta \alpha$ & 2 & 313.0 & 156.5 & 9.88 & 0.0029 \\
\hline
\end{tabular}

GL. Grados de libertad 
De acuerdo con la literatura, los iones oxalato juegan un papel muy importante en la fotodegradación de compuestos orgánicos asistida con ferrioxalato ya que favorecen la continua regeneración de iones $\mathrm{Fe}^{2+}$ vía fotorreducción con la luz incidente y la generación extra de radicales libres (principalmente $\mathrm{OH}^{*}$ ) por la fotoquímica del ferrioxalato (Safarzadeh et al. 1997). Además, en este sistema las principales especies fotoactivas formadas son $\mathrm{Fe}(\mathrm{OH})^{2+}$, $\mathrm{Fe}\left(\mathrm{C}_{2} \mathrm{O}_{4}\right)^{+}, \mathrm{Fe}\left(\mathrm{C}_{2} \mathrm{O}_{4}\right)^{2-}$ y $\mathrm{Fe}\left(\mathrm{C}_{2} \mathrm{O}_{4}\right)_{3}^{3-}$, de las cuales de acuerdo con la literatura las dos últimas son más activas fotolíticamente y se favorecen a concentraciones elevadas de ácido oxálico según las ecuaciones 23 a 25 (Safarzadeh et al. 1997, Jeong et al. 2005, Monteagudo et al. 2010).

$$
\begin{aligned}
& \mathrm{Fe}^{3+}+\mathrm{C}_{2} \mathrm{O}_{4}^{2-} \rightleftarrows \mathrm{Fe}\left(\mathrm{C}_{2} \mathrm{O}_{4}\right)^{+} \\
& \mathrm{Fe}\left(\mathrm{C}_{2} \mathrm{O}_{4}\right)^{+}+\mathrm{C}_{2} \mathrm{O}_{4}^{2-} \rightleftarrows \mathrm{Fe}\left(\mathrm{C}_{2} \mathrm{O}_{4}\right)_{2}^{-} \\
& \mathrm{Fe}\left(\mathrm{C}_{2} \mathrm{O}_{4}\right)_{2}^{-}+\mathrm{C}_{2} \mathrm{O}_{4}^{2-} \rightleftarrows \mathrm{Fe}\left(\mathrm{C}_{2} \mathrm{O}_{4}\right)_{3}^{3-}
\end{aligned}
$$

Por otro lado, el efecto adverso con el incremento en la concentración inicial de $\mathrm{Fe}^{2+}$ se debe posiblemente dos razones: a que la cantidad de iones $\mathrm{Fe}^{2+}$ excede a la necesaria para formar la cantidad óptima del complejo ferrioxalato y por lo tanto, éste se precipita en la especie insoluble y poco activa $\mathrm{Fe}(\mathrm{OH})_{3}$ (Monteagudo et al. 2012) la cual, además, disminuye la penetración de la luz a través de la solución irradiada y por lo tanto se producen menos radicales reactivos hidroperóxido (Selvam et al. 2005). Por otro lado, altas concentraciones de $\mathrm{Fe}^{2+}$ pueden inhibir el proceso debido a que este ion compite con el $\mathrm{Fe}^{3+}$ por los diferentes radicales para formar los intermediarios (ecuaciones 26 a 28) (Aguirre 2010, Monteagudo et al. 2010).

$$
\begin{aligned}
& \mathrm{Fe}(\mathrm{II})+\mathrm{HO}^{\cdot} \rightarrow \mathrm{Fe}(\mathrm{II})+\mathrm{OH}^{-} \\
& \mathrm{Fe}(\mathrm{II})+\mathrm{HO}_{2} \rightarrow \mathrm{Fe}(\mathrm{III})\left(\mathrm{HO}_{2}\right)^{2+} \\
& \mathrm{Fe}(\mathrm{III})+\mathrm{O}_{2}^{--}+\mathrm{H}^{+} \rightarrow \mathrm{Fe}(\mathrm{III})\left(\mathrm{HO}_{2}\right)^{2+}
\end{aligned}
$$

En la misma figura 3 se observa que los perfiles del porcentaje de degradación de etilenglicol, en función de la relación molar $\mathrm{Fe}^{2+} / \mathrm{C}_{2} \mathrm{O}_{4}^{2-}$, son similares a diferentes concentraciones iniciales de $\mathrm{H}_{2} \mathrm{O}_{2}$. No obstante, se obtuvieron mayores porcentajes de degradación al aumentar la concentración inicial de $\mathrm{H}_{2} \mathrm{O}_{2}$. Este incremento es debido a que el $\mathrm{H}_{2} \mathrm{O}_{2}$ genera radicales hidroxilo adicionales responsables de la degradación oxidativa y en algunos casos de la mineralización de muchas sustancias químicas en el sistema $\mathrm{H}_{2} \mathrm{O}_{2} / \mathrm{UV}$ asistido con ferrioxalato (ecuaciones 4 y 13 a 17) (Lee et al. 2003).

La figura 4 muestra el porcentaje de consumo de $\mathrm{H}_{2} \mathrm{O}_{2}$ después de $3 \mathrm{~h}$ de irradiación en función de la relación molar $\mathrm{Fe}^{2+} / \mathrm{C}_{2} \mathrm{O}_{4}^{2-}$ a diferentes concentraciones iniciales de $\mathrm{H}_{2} \mathrm{O}_{2}$. Los resultados demuestran que la concentración de $\mathrm{H}_{2} \mathrm{O}_{2}$ disminuye rápidamente, alcanzándose porcentajes de consumo del mismo mayores al $40 \%$ en los dos casos.

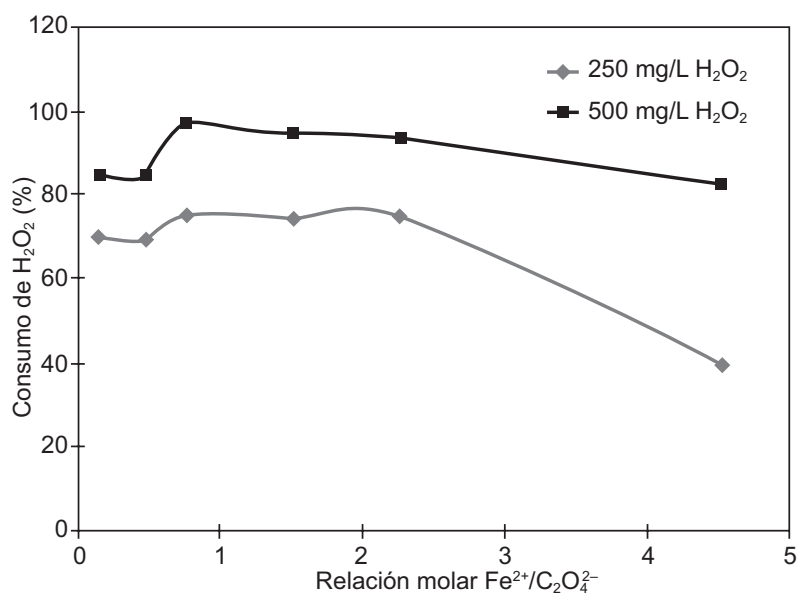

Fig. 4. Porcentaje de consumo de $\mathrm{H}_{2} \mathrm{O}_{2}$ después de $3 \mathrm{~h}$ de irradiación en función de la relación molar $\mathrm{Fe}^{2+} / \mathrm{C}_{2} \mathrm{O}_{4}^{2-}$ a diferentes concentraciones iniciales de $\mathrm{H}_{2} \mathrm{O}_{2}$

El pH es un factor importante en los sistemas fotoFenton asistido con ferrioxalato, ya que éste puede afectar directamente la especiación, solubilidad del hierro y la producción de radicales activos para la degradación de los compuestos orgánicos (Joonseon y Yoon 2005, Aguirre 2010, Monteagudo et al. 2010, Qing et al. 2011). En la figura 5 se puede observar que el valor final del $\mathrm{pH}$ del medio de reacción es muy similar para las diferentes relaciones molares $\mathrm{Fe}^{2+}$ / $\mathrm{C}_{2} \mathrm{O}_{4}^{2-}$ a distintas concentraciones iniciales de $\mathrm{H}_{2} \mathrm{O}_{2}$. $\mathrm{El} \mathrm{pH}$ se mantuvo entre 2.5 y 3.0 durante todas las reacciones. Como se mencionó anteriormente, en el presente sistema se pueden formar diferentes especies fotoactivas como $\mathrm{Fe}(\mathrm{OH})^{2+}, \mathrm{Fe}\left(\mathrm{C}_{2} \mathrm{O}_{4}\right)^{+}, \mathrm{Fe}\left(\mathrm{C}_{2} \mathrm{O}_{4}\right)_{2}{ }^{-} \mathrm{y}$ $\mathrm{Fe}\left(\mathrm{C}_{2} \mathrm{O}_{4}\right)_{3}^{-3}$ (Aguirre 2010, Araña et al. 2012), siendo la última especie una de las más fotolíticamente activas. La formación de dichas especies es función del $\mathrm{pH}$ del medio de reacción y de las concentraciones de $\mathrm{Fe}^{2+}, \mathrm{Fe}^{3+}, \mathrm{C}_{2} \mathrm{O}_{4}^{2-}$ y $\mathrm{H}_{2} \mathrm{O}_{2}$ (ecuaciones 23 a 25).

Teniendo en cuenta que bajo las condiciones de reacción utilizadas la mayoría del hierro presente en el sistema está en forma de complejos hierro-oxalato, se decidió simular los diagramas de especiación para los diferentes complejos de 


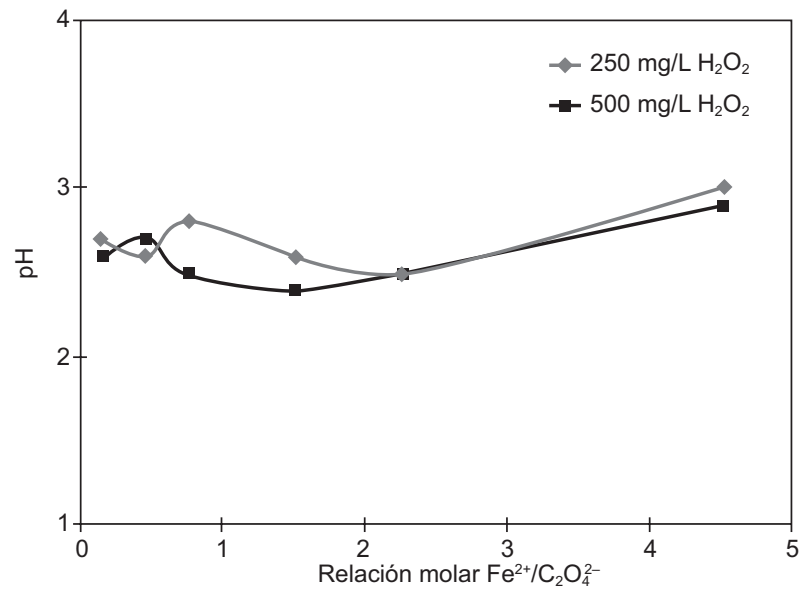

Fig. 5. Valor final del pH de la solución de reacción después de $3 \mathrm{~h}$ de irradiación en función de la relación molar $\mathrm{Fe}^{2+} /$ $\mathrm{C}_{2} \mathrm{O}_{4}^{2-}$ a diferentes concentraciones iniciales de $\mathrm{H}_{2} \mathrm{O}_{2}$

ferrioxalato utilizando el programa Medusa-Hydra chemical equilibrium, para identificar el tipo de especies formadas bajo las condiciones de reacción utilizadas en la presente investigación.

En el cuadro IV se muestra la fracción aproximada de cada una de las especies formadas a las diferentes concentraciones totales de $\mathrm{Fe}^{3+}$ y $_{2} \mathrm{O}_{4}^{2-}$ en un intervalo de $\mathrm{pH}$ entre 2.5 y 3.0 con el porcentaje de degradación de etilenglicol obtenido en cada caso. Como se puede observar, el porcentaje de degradación de etilenglicol depende fuertemente del tipo y la cantidad de especies formadas.

Por ejemplo, la fracción de $\mathrm{Fe}(\mathrm{OH})^{2+}$ es insignificante en todos los casos y los iones ferrioxalato $\mathrm{Fe}\left(\mathrm{C}_{2} \mathrm{O}_{4}\right)^{+}, \mathrm{Fe}\left(\mathrm{C}_{2} \mathrm{O}_{4}\right)_{2}^{-}$y $\mathrm{Fe}\left(\mathrm{C}_{2} \mathrm{O}_{4}\right)_{3}^{3-}$ constituyen las principales especies de $\mathrm{Fe}^{3+}$ presentes en el sistema.
Por otro lado, a valores de $\mathrm{pH}$ entre 2.5 y 3.0 se encuentran especies como $\mathrm{Fe}\left(\mathrm{C}_{2} \mathrm{O}_{4}\right)_{2}^{-}, \mathrm{Fe}\left(\mathrm{C}_{2} \mathrm{O}_{4}\right)^{+}$, $\mathrm{Fe}\left(\mathrm{C}_{2} \mathrm{O}_{4}\right)_{3}^{3-}$ y $\mathrm{Fe}_{2} \mathrm{O}_{3}$, sin embargo, se puede observar que las condiciones bajo las cuales se obtuvo una mayor proporción de las especies $\mathrm{Fe}\left(\mathrm{C}_{2} \mathrm{O}_{4}\right)_{2}^{-} \mathrm{y}$ $\mathrm{Fe}\left(\mathrm{C}_{2} \mathrm{O}_{4}\right)_{3}^{3-}$ corresponden a los mayores porcentajes de remoción de etilenglicol, mientras que a bajos porcentajes de degradación predominó la especie insoluble de hierro $\left(\mathrm{Fe}_{2} \mathrm{O}_{3}\right)$.

Además, también se puede observar que aunque se obtenga una fracción de complejos de ferrioxalato similar, la degradación de etilenglicol varía de acuerdo con la concentración inicial del $\mathrm{H}_{2} \mathrm{O}_{2}$. Lo cual se puede deber a la presencia de especies $\mathrm{O}_{2}^{--}$y $\mathrm{HO}_{2}$, las cuales también son sensibles a los valores de $\mathrm{pH}$ del medio (ecuaciones 29 y 30 ) y al reaccionar con el $\mathrm{Fe}^{2+}$ constituyen la principal fuente de $\mathrm{H}_{2} \mathrm{O}_{2}$ en el sistema foto/ferrioxalato (ecuaciones 32 y 33 ) (Joonseon y Yoon 2005, Prato 2007).

$$
\begin{aligned}
& \mathrm{HC}_{2} \mathrm{O}_{4}^{+} \rightleftarrows \mathrm{C}_{2} \mathrm{O}_{4}^{2-}+\mathrm{H}^{+} \\
& \mathrm{HO}_{2} \rightleftarrows \mathrm{O}_{2}^{--}+\mathrm{H}^{+} \\
& \mathrm{Fe}^{2+}+\mathrm{O}_{2}^{--} \rightarrow \mathrm{Fe}^{3+}+\mathrm{H}_{2} \mathrm{O}_{2}+\mathrm{OH}^{-} \\
& \mathrm{Fe}^{2+}+\mathrm{HO}_{2}^{-} \rightarrow \mathrm{Fe}^{3+}+\mathrm{H}_{2} \mathrm{O}_{2}+\mathrm{OH}^{-}
\end{aligned}
$$

Los resultados obtenidos demuestran que las condiciones óptimas para la degradación de un $90 \%$ de etilenglicol después de $3 \mathrm{~h}$ de irradiación artificial consisten en $10 \mathrm{mg} / \mathrm{L}$ de $\mathrm{Fe}^{2+}, 150 \mathrm{mg} / \mathrm{L}$ de $\mathrm{C}_{2} \mathrm{O}_{4}^{2-} \mathrm{y}$ $500 \mathrm{mg} / \mathrm{L}$ de $\mathrm{H}_{2} \mathrm{O}_{2}$. Como se muestra en la figura 6, el proceso involucró predominantemente especies

\begin{tabular}{|c|c|c|c|c|c|}
\hline \multicolumn{4}{|c|}{ Fracción molar } & \multirow{2}{*}{$\begin{array}{c}{\left[\mathrm{H}_{2} \mathrm{O}_{2}\right]_{0}} \\
(\mathrm{mg} / \mathrm{L})\end{array}$} & \multirow{2}{*}{$\begin{array}{c}\text { Degradación } \\
\text { etilenglicol }(\%)\end{array}$} \\
\hline $\mathrm{Fe}\left(\mathrm{C}_{2} \mathrm{O}_{4}\right)_{2}^{-}$ & $\mathrm{Fe}\left(\mathrm{C}_{2} \mathrm{O}_{4}\right)^{+}$ & $\mathrm{Fe}\left(\mathrm{C}_{2} \mathrm{O}_{4}\right)_{3}^{3-}$ & $\mathrm{Fe}_{2} \mathrm{O}_{3}$ & & \\
\hline 0.82 & 0.12 & 0.06 & 0 & 250 & 63 \\
\hline 0.82 & 0.12 & 0.06 & 0 & 500 & 85 \\
\hline 0.84 & 0.01 & 0.15 & 0 & 250 & 67 \\
\hline 0.84 & 0.01 & 0.15 & 0 & 500 & 90 \\
\hline 0.18 & 0.02 & 0.03 & 0.77 & 250 & 51 \\
\hline 0.18 & 0.02 & 0.03 & 0.77 & 500 & 41 \\
\hline 0.59 & 0.03 & 0.12 & 0.26 & 250 & 52 \\
\hline 0.59 & 0.03 & 0.12 & 0.26 & 500 & 78 \\
\hline 0.1 & 0.07 & 0 & 0.83 & 250 & 30 \\
\hline 0.1 & 0.07 & 0 & 0.83 & 500 & 39 \\
\hline 0.3 & 0.03 & 0.02 & 0.65 & 250 & 48 \\
\hline 0.3 & 0.03 & 0.02 & 0.65 & 500 & 71 \\
\hline
\end{tabular}
$\mathrm{Fe}\left(\mathrm{C}_{2} \mathrm{O}_{4}\right)_{2}^{-}$y $\mathrm{Fe}\left(\mathrm{C}_{2} \mathrm{O}_{4}\right)_{3}^{3-}$, las cuales permiten una

CUADRO IV. FRACCIÓN MOLAR DE LAS ESPECIES FORMADAS CON $0.18 \mathrm{mM} \mathrm{DE} \mathrm{Fe}^{3+} \mathrm{Y} 1.20 \mathrm{mM} \mathrm{DE}_{2} \mathrm{O}_{4}^{2-} \mathrm{A}$ UN pH ENTRE 2.5-3.0 


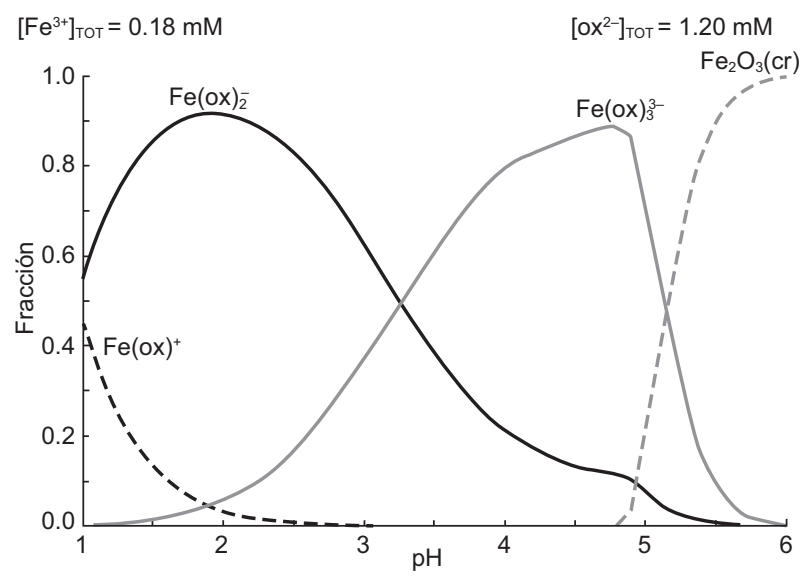

Fig. 6. Especiación de complejos de ferrioxalato calculado por Medusa en un intervalo de $\mathrm{pH}$ entre 1 y 6 bajo las mejores condiciones de reacción: $\left[\mathrm{Fe}^{3+}\right]_{\text {Total }}=10 \mathrm{mg} / \mathrm{L} \mathrm{y}\left[\mathrm{C}_{2} \mathrm{O}_{4}^{2-}\right]$ Total $=150 \mathrm{mg} / \mathrm{L}$

formación más eficiente de radicales hidroxilo en presencia de oxígeno disuelto bajo radiación UV. Esto demuestra que la eficiencia de degradación de etilenglicol es mucho más alta en el sistema ferrioxalato que en el sistema $\mathrm{Fe}$ (III)-hidroxo a $\mathrm{pH}$ entre 2.5 y 3.0. Esto es consistente con el hecho de que los complejos de ferrioxalato tienen mayor fotoactividad que el $\mathrm{Fe}(\mathrm{OH})^{2+}$, como se ha mencionado en trabajos previos (Joonseon y Yoon 2005).

La figura 7 muestra la variación del porcentaje de degradación de etilenglicol y del $\mathrm{pH}$ del medio en función del tiempo bajo las mejores condiciones de reacción: $\left[\mathrm{Fe}^{3+}\right]=10 \mathrm{mg} / \mathrm{L},\left[\mathrm{C}_{2} \mathrm{O}_{4}^{2-}\right]=150 \mathrm{mg} / \mathrm{L}$ y $\left[\mathrm{H}_{2} \mathrm{O}_{2}\right]=500 \mathrm{mg} / \mathrm{L}$. Como se observa, el sistema propuesto favorece la formación de complejos de

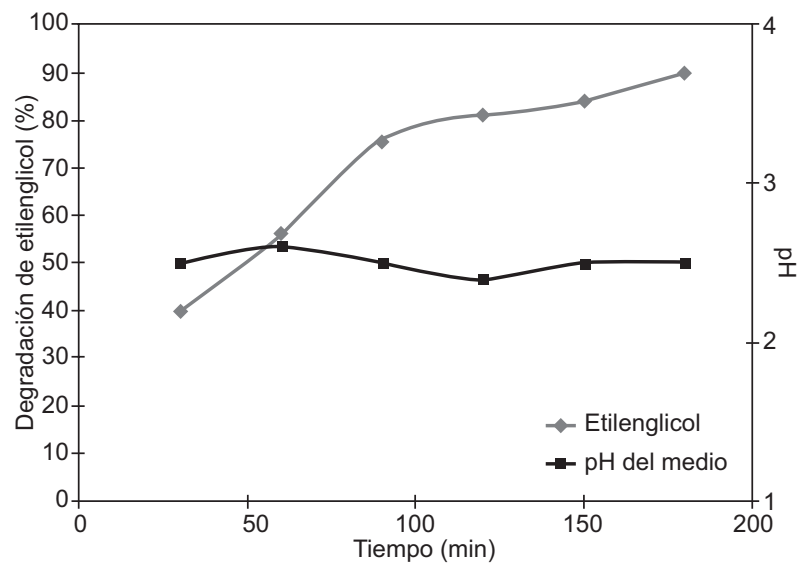

Fig. 7. Variación del porcentaje de degradación de etilenglicol y del $\mathrm{pH}$ de la solución en función del tiempo bajo las mejores condiciones de reacción. $\left[\mathrm{Fe}^{3+}\right]=10 \mathrm{mg} / \mathrm{L}$, $\left[\mathrm{C}_{2} \mathrm{O}_{4}^{2-}\right]=150 \mathrm{mg} / \mathrm{L}$ y $\left[\mathrm{H}_{2} \mathrm{O}_{2}\right]=500 \mathrm{mg} / \mathrm{L}$ ferrioxalato, lo que mantiene estable el valor del $\mathrm{pH}$ del medio (Monteagudo et al. 2012). Esto permite la degradación rápida y eficiente del etilenglicol. Por otro lado, la figura 8 muestra la concentración de los compuestos intermediarios identificados en función del tiempo bajo las mejores condiciones de reacción. El etilenglicol fue convertido a ácido acético y ácido fórmico resultando en una pérdida de demanda química de oxígeno (McGinnis et al. 2001).

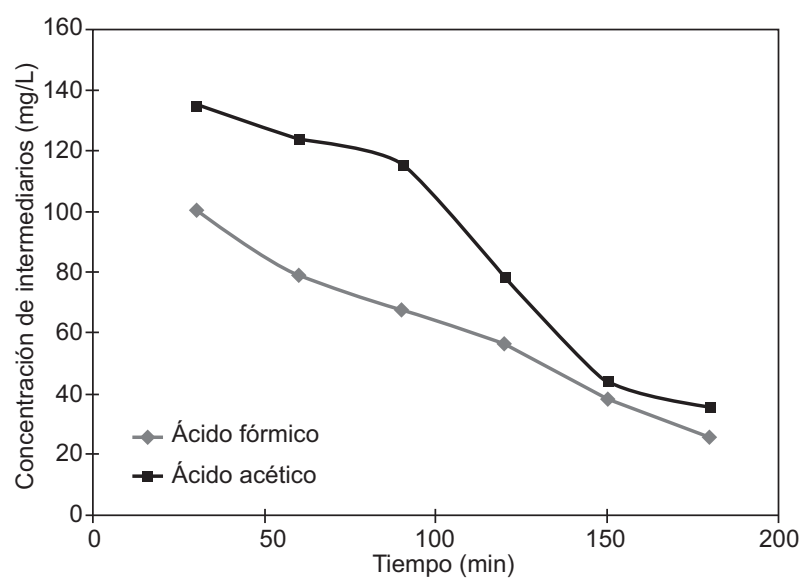

Fig. 8. Concentración de los compuestos intermediarios identificados en función del tiempo bajo las mejores condiciones de reacción. $\left[\mathrm{Fe}^{3+}\right]=10 \mathrm{mg} / \mathrm{L},\left[\mathrm{C}_{2} \mathrm{O}_{4}^{2-}\right]=150 \mathrm{mg} / \mathrm{L} \mathrm{y}$ $\left[\mathrm{H}_{2} \mathrm{O}_{2}\right]=500 \mathrm{mg} / \mathrm{L}$

Adicionalmente, una remoción del $85 \%$ del COT obtenido del análisis de la solución final después de degradación bajo las mejores condiciones de reacción, permite comprobar que la mayor parte de los intermediarios o productos de reacción se encuentran en cantidades traza, lo cual demuestra la alta eficiencia del tratamiento para lograr niveles altos tanto en la degradación como en la mineralización de etilenglicol presente en una solución ideal.

\section{Degradación del etilenglicol presente en un efluen- te real bajo las condiciones óptimas}

Las características fisicoquímicas del efluente original colectado en la empresa de pinturas se muestran en el cuadro V. La concentración de etilenglicol encontrada corresponde a $6500 \mathrm{mg} / \mathrm{L}$, dicho valor está en el intervalo de concentraciones (1000-25 $000 \mathrm{mg} / \mathrm{L})$ encontrado en aguas residuales contaminadas con este disolvente (Araña et al. 2012). Adicionalmente, se ha reportado que concentraciones de etilenglicol similares a las encontradas en el presente estudio pueden tener efectos negativos en 
CUADRO V. CARACTERÍSTICAS FISICOQUÍMICAS DEL EFLUENTE ORIGINAL

\begin{tabular}{|c|c|c|}
\hline Parámetro & Unidad & Valor \\
\hline Temperatura & ${ }^{\circ} \mathrm{C}$ & 23.3 \\
\hline Color & UPC & 90 \\
\hline Turbiedad & UNT & 650 \\
\hline Conductividad & $\mathrm{mS} / \mathrm{cm}$ & 140 \\
\hline $\mathrm{pH}$ & Unidades de $\mathrm{pH}$ & 8.9 \\
\hline Oxígeno disuelto & $\mathrm{mg} \mathrm{O}_{2} / \mathrm{L}$ & 1.57 \\
\hline $\begin{array}{l}\text { Demanda química de } \\
\text { oxígeno (DQO) }\end{array}$ & $\mathrm{mg} \mathrm{O}_{2} / \mathrm{L}$ & 43000 \\
\hline $\begin{array}{l}\text { Demanda bioquímica de } \\
\text { oxígeno }\left(\mathrm{DBO}_{5}\right)\end{array}$ & $\mathrm{mg} \mathrm{O}_{2} / \mathrm{L}$ & 25000 \\
\hline Carbono orgánico total (COT) & $\mathrm{mg} / \mathrm{L}$ & 15000 \\
\hline Relación $\mathrm{DBO}_{5} / \mathrm{DQO}$ & Adimensional & 0.58 \\
\hline Relación $\mathrm{DBO}_{5} / \mathrm{COT}$ & Adimensional & 1.67 \\
\hline Sólidos sedimentales & $\mathrm{mL} / \mathrm{mL}$ & 0.4 \\
\hline Sólido disueltos & $\mathrm{mg} / \mathrm{L}$ & 60 \\
\hline Sólidos totales & $\mathrm{mg} / \mathrm{L}$ & 2 \\
\hline Etilenglicol & $\mathrm{mg} / \mathrm{L}$ & 6500 \\
\hline
\end{tabular}

DQO: demanda química de oxígeno, $\mathrm{DBO}_{5}$ : demanda bioquímica de oxígeno, COT: carbono orgánico total.

los ecosistemas acuáticos. Por ejemplo, estudios de toxicidad de etilenglicol muestran que concentraciones del mismo por encima de $3000 \mathrm{mg} / \mathrm{L}$ en el agua producen inhibición en el crecimiento de plantas acuáticas como Lemna minor. Otros autores han indicado que la toxicidad de este solvente para microorganismos está por encima de $1000 \mathrm{mg} / \mathrm{L}$ (Rivas et al. 2002). Asimismo, se ha reportado que concentraciones de etilenglicol de $6500 \mathrm{mg} / \mathrm{L}$ son letales para el crecimiento de cierto tipo de algas (Araña et al. 2012).

Uno de los indicadores más importantes de la calidad del agua es el oxígeno disuelto (OD), el cual corresponde a $1.57 \mathrm{mg} / \mathrm{L}$ para el agua recolectada inicialmente. Este valor se encuentra muy por debajo de los valores normales de oxígeno establecidos para aguas naturales $(7.0-8.0 \mathrm{mg} / \mathrm{L})$, lo que indica contaminación apreciable de naturaleza orgánica, confirmada por los altos valores obtenidos para la $\mathrm{DBO}_{5}$ y el COT. Por otra parte, un parámetro generalmente
CUADRO VI. CRITERIOS DE BIODEGRADABILIDAD SEGÚN LA RELACIÓN DBO $5 /$ DQO

\begin{tabular}{cl}
\hline $\mathrm{DBO}_{5} / \mathrm{DQO}$ & Carácter \\
\hline$>0.8$ & Muy biodegradable \\
$0.7-0.8$ & Biodegradable \\
$0.3-0.7$ & Poco biodegradable \\
$<0.3$ & No biodegradable \\
\hline
\end{tabular}

$\mathrm{DBO}_{5}$ : demanda bioquímica de oxígeno, DQO: demanda química de oxígeno

utilizado para identificar la biodegradabilidad de los diversos tipos de vertidos es la relación de biodegradabilidad $\mathrm{DBO}_{5} / \mathrm{DQO}$, la cual permite determinar qué cantidad de la DQO (materia orgánica e inorgánica contenida en una muestra) de un vertido industrial es susceptible de ser depurada por los microorganismos en 5 días $\left(\mathrm{DBO}_{5}\right)$. En el cuadro VI se especifican algunos criterios que permiten determinar el carácter de biodegradabilidad de los diferentes vertidos (Ardila et al. 2012).

Según la relación $\mathrm{DBO}_{5} / \mathrm{DQO}$ obtenida para el agua residual original $(0.58)$, se puede afirmar que este efluente es de naturaleza poco biodegradable; es decir, la mayor cantidad de DQO presente en el vertido no se puede oxidar bioquímicamente. Además, de acuerdo con la literatura (Aziz y Tebbutt 1980), un valor para la relación $\mathrm{DBO}_{5} / \mathrm{COT}>1.44$, es un indicativo de que el efluente tiene alto contenido de compuestos orgánicos de carácter no biodegradable. Por lo tanto, un tratamiento biológico no sería suficiente para remover la materia orgánica de carácter recalcitrante presente en el presente vertido industrial. Estos datos son una evidencia más de la necesidad de implementar un sistema para la remoción de etilenglicol previo a la descarga.

Para evaluar las condiciones óptimas encontradas en la degradación de $1000 \mathrm{mg} / \mathrm{L}$ de etilenglicol en agua destilada, se diluyó una muestra de agua residual real hasta obtener la misma concentración del contaminante. Como se muestra en el cuadro VII, en este caso se degradó un $85 \%$ del mismo $(\sim 850 \mathrm{mg} / \mathrm{L})$,

CUADRO VII. PORCENTAJES DE REMOCIÓN DE ETG, DQO, DBO 5 Y COT DEL AGUA RESIDUAL REAL

\begin{tabular}{|c|c|c|c|c|c|c|}
\hline \multirow{2}{*}{$\begin{array}{l}\text { ETG inicial en el agua residual } \\
\qquad(\mathrm{mg} / \mathrm{L})\end{array}$} & \multicolumn{4}{|c|}{ Remoción (\%) } & \multirow{2}{*}{$\mathrm{DBO}_{5} / \mathrm{DQO}$} & \multirow{2}{*}{$\mathrm{DBO}_{5} / \mathrm{COT}$} \\
\hline & ETG & DQO & $\mathrm{DBO}_{5}$ & COT & & \\
\hline 1000 & 85 & 40 & 55 & 67 & 0.80 & 1.37 \\
\hline 6500 & 18 & 35 & 43 & 52 & 0.71 & 1.38 \\
\hline
\end{tabular}

ETG: etilenglicol, DQO: demanda química de oxígeno, $\mathrm{DBO}_{5}$ : demanda bioquímica de oxígeno, COT: carbono orgánico total 
dicho valor es muy cercano al obtenido en el tratamiento de la muestra de etilenglicol en agua (90\%), lo que indica la ausencia de efectos inhibidores debidos a la presencia de otros contaminantes. Adicionalmente, se identificaron concentraciones de ácido acético y ácido fórmico menores a $106 \mathrm{mg} / \mathrm{L}$, lo que indica una acumulación baja de productos intermediarios provenientes de la degradación de etilenglicol.

El seguimiento del COT, DQO y $\mathrm{DBO}_{5}$ en este tipo de tecnologías oxidativas, es una herramienta importante porque la disminución en el valor de estos parámetros puede garantizar que durante el proceso no se acumulan contaminantes recalcitrantes o intermediarios de mayor persistencia o toxicidad que los iniciales (McGinnis et al. 2000). Además, la evaluación cuidadosa de las relaciones $\mathrm{DBO}_{5} / \mathrm{DQO}$ y $\mathrm{DBO}_{5} / \mathrm{COT}$ permite determinar la calidad de biodegradabilidad del efluente después del tratamiento. Como se observa en el cuadro VII, también se lograron porcentajes de remoción de $\mathrm{DQO}, \mathrm{DBO}_{5}$ y COT iguales a 40, 55 y $67 \%$, respectivamente. Además, de acuerdo con los valores obtenidos para las relaciones $\mathrm{DBO}_{5} / \mathrm{COT}(1.37)$ y $\mathrm{DBO}_{5} / \mathrm{DQO}(0.80)$, el efluente final es de naturaleza biodegradable. Estos resultados confirman que las condiciones optimizadas son adecuadas para degradar $\sim 1000 \mathrm{mg} / \mathrm{L}$ de etilenglicol presente incluso en un agua residual real. Además el tratamiento aplicado facilitó la oxidación de materia orgánica adicional biodegradable y no biodegradable presente en el efluente.

Por otro lado, pruebas realizadas con el agua residual original conteniendo $6500 \mathrm{mg} / \mathrm{L}$ de etilenglicol mostraron un porcentaje de degradación del mismo de tan sólo el 18\% (Cuadro VII). Lo cual indica que las condiciones óptimas encontradas no son las adecuadas para degradar las $6500 \mathrm{mg} / \mathrm{L}$ de etilenglicol presente en dicha agua residual. De esta forma, se considera que probablemente las cantidades de los iones oxalato y ferroso y del peróxido de hidrógeno optimizadas son insuficientes para degradar completamente dicho contaminante. Este hecho es muy probable debido a que el $\mathrm{Fe}$ responsable de la producción de radicales $\mathrm{OH}^{*}$ no es un catalizador sino que se consume durante la reacción, además la cantidad de radicales $\mathrm{OH}^{*}$ presentes en el sistema también depende de la cantidad de $\mathrm{H}_{2} \mathrm{O}_{2}$ adicionada al mismo.

\section{CONCLUSIONES}

Las variables investigadas, concentración inicial de $\mathrm{Fe}^{2+}, \mathrm{C}_{2} \mathrm{O}_{4}^{2-}$ y $\mathrm{H}_{2} \mathrm{O}_{2}$, afectan la eficiencia de mineralización del etilenglicol.
Los mayores niveles demineralización de $1000 \mathrm{mg} / \mathrm{L}$ de etilenglicol fueron 90 y $85 \%$, para el agua destilada y el agua residual real, respectivamente. Se obtuvieron después de $3 \mathrm{~h}$ de irradiación artificial a una longitud de onda de $\sim 365 \mathrm{~nm}$, utilizando $10 \mathrm{mg} / \mathrm{L}$ de $\mathrm{Fe}^{2+}, 150 \mathrm{mg} / \mathrm{L}$ de $\mathrm{C}_{2} \mathrm{O}_{4}^{2-}$ y $500 \mathrm{mg} / \mathrm{L}$ de $\mathrm{H}_{2} \mathrm{O}_{2}$.

El etilenglicol fue convertido a ácido acético y ácido fórmico en cantidades traza $(<136 \mathrm{mg} / \mathrm{L})$, resultando en una pérdida de demanda química de oxígeno. Ningún otro producto intermediario fue detectado. El proceso involucró predominantemente especies de Fe (III), comúnmente llamadas complejos de ferrioxalato. Estas fueron $\mathrm{Fe}\left(\mathrm{C}_{2} \mathrm{O}_{4}\right)_{2}^{-}$y $\mathrm{Fe}\left(\mathrm{C}_{2} \mathrm{O}_{4}\right)_{3}^{3-}$, las cuales permiten la formación de radicales hidroxilo en presencia de peróxido de hidrógeno bajo radiación UV-Vis.

Las cantidades óptimas de ion oxalato, ion ferroso y peróxido de hidrógeno, dependen la concentración de etilenglicol a degradar, por lo tanto, es importante optimizar estos parámetros para cada concentración de dicho contaminante.

\section{AGRADECIMIENTOS}

Los autores agradecen al Politécnico Colombiano Jaime Isaza Cadavid y a la Universidad Autónoma Metropolitana-Iztapalapa de México por la financiación del presente proyecto de investigación.

\section{REFERENCIAS}

Aguirre M. (2010). Degradación catalítica de contaminantes orgánicos mediante procesos foto-fenton UV-A/C/solar asistidos con ferrioxalato. Tesis de Doctorado. Departamento de Ingeniería Química. Universidad de Castilla-La Mancha. Ciudad real, España, 392 pp.

Andreozzi R., Caprio V., Insola A. y Marotta R. (1999). Advanced oxidation processes (AOP) for water purification and recovery. Catal. Today. 53, 51-59. DOI:10.1016/S0920-5861(99)00102-9.

Araña J., Ortega M. J. A., Herrera M. J. A., Doña R. J. M., González D. O. y Pérez P. J. (2012). Thermal effect of carboxylic acids in the degradation by photoFenton of high concentrations of ethylene glycol. Appl. Catal. B: Environ. 113-114, 107-115. DOI:10.1016/j. apcatb.2011.11.025.

Ardila A. A. N., Reyes C. J., Arriola V. E. y Hernández J. A. (2012). Remoción fotocatalítica de DQO, DBO5 y COT de efluentes de la industria farmacéutica. Revista Politécnica 15, 9-17. 
Aziz J. y Tebbutt T., (1980). Significance of COD, BOD and TOC correlations in kinetic models of biological oxidation. Water Res. 14, 319-324. DOI:10.1016/00431354(80)90077-9.

García E. F., Féliz M. R. y Capparelli A. L. (1997). Effect of temperature on hydrogen peroxide photolysis in aqueous solutions. J. Photochem. Photobiol. A Chem. 110, 235-242. DOI:10.1016/S1010-6030(97)00183-4.

Ghiselli G., Jardim W. F., Litter M. I. y Mansilla. H. D. (2004). Destruction of EDTA using Fenton and photoFenton-like reactions under UV-A irradiation. J. Photochem. Photobiol. A Chem.167, 59-67. DOI:10.1016/j. jphotochem.2004.02.005.

Giroto J. A., Teixeira A. C. S. C., Nascimento C. A. O. y Guardani R. (2010). Degradation of poly(ethylene glycol) in aqueous solution by photo-Fenton and $\mathrm{H}_{2} \mathrm{O}_{2} /$ UV processes. Ind. Eng. Chem. Res. 49, 3200-3206. DOI:10.1021/ie9015792.

Joonseon J. y Yoon J. (2005). pH effect on OH radical production in photo/ferrioxalate system. Water Res. 39, 2893-2900. DOI:10.1016/j.watres.2005.05.014.

Kurt, U., Avsar Y. y Gonullu M. T. (2006). Treatability of water-based paint wastewater with Fenton process in different reactor types. Chemosphere 64, 1536-1540. DOI:10.1016/j.chemosphere.2005.11.026.

Kusic H., Koprivanac N., Loncaric B. A. y Selanec. I. (2006). Photo-assisted Fenton type processes for the degradation of phenol: A kinetic study. J. Hazard. Mater. 136, 632-644. DOI:10.1016/j.jhazmat.2005.12.046.

Lee Y., Jeong J., Lee C., Kim S. y Yoon J. (2003). Influence of various reaction parameters on 2,4-D removal in photo/ferrioxalate $/ \mathrm{H}_{2} \mathrm{O}_{2}$ process. Chemosphere 51, 901-912. DOI:10.1016/S0045-6535(03)00044-4.

Machulek Jr. A., Quina F. H., Gozzi F., Volnir O. S., Friedrich L. C. y. Moraes J. E. F. (2012). Fundamental mechanistic studies of the photo-Fenton reaction for the degradation of organic pollutants. En: Organic pollutants ten years after the stockholm convention-environmental and analytical update. (Dr. Tomasz Puzyn, Ed.), InTech, Shanghai, China. pp. 271-292. DOI: 10.5772/30995.

McGinnis B. D., Adams V. D. y Middlebrooks E. J. (2000). Degradation of ethylene glycol in photo Fenton systems. Water Res. 34, 2346-2354. DOI:10.1016/S00431354(99)00387-5.

McGinnis B. D., Adams V. D. y Middlebrooks E. J. (2001). Degradation of ethylene using Fenton's reagent and UV. Chemosphere 45, 101-108. DOI:10.1016/S00456535(00)00597-X.

Monteagudo J. M., Durán A., Corral J. M., Carnicer A., Frades. J. M. y Alonso M. A. (2012). Ferrioxalateinduced solar photo-Fenton system for the treatment of winery wastewaters. Chem. Eng. J. 181-182, 281-288. DOI:10.1016/j.cej.2011.11.080.
Monteagudo J.M., Durán A., San Martín I. y Aguirre M. (2010). Catalytic degradation of Orange II in a ferrioxal ate-assisted photo-Fenton process using a combined UV-A/C-solar pilot-plant system Appl. Catal. B: Environ. 95, 120-129. DOI:10.1016/j.apcatb.2009.12.018.

Nogueira R. F. P., Silva M. R. A. y Silva A. G. X. (2005). Influence of the iron source on the solar photo-Fenton degradation of different classes of organic compounds. Sol. Energy. 79, 384-392. DOI:10.1016/j. solener.2005.02.019.

Prato G. D. (2007). Tratamiento de aguas residuales industriales fenólicas sintéticas mediante procesos avanzados de oxidación. Tesis de Maestría en Ciencias en Ingeniería Química. Universidad Iberoamericana. México, D.F., 148 pp.

Prato G. D., Vasquez M. R. y Hernandez E. M. (2009). Solar photoassisted advanced oxidation of synthetic phenolic wastewaters using ferrioxalate complexes. Sol. Energy. 83, 306-315. DOI:10.1016/j.solener.2008.08.005.

Qing L., Liu H., Li F., Zeng F. y Liu C. (2011). Effect of $\mathrm{pH}$ on pentachlorophenol degradation in irradiated iron/oxalate systems. Chem. Eng. J. 168, 1209-1216. DOI:10.1016/j.cej.2011.02.017.

Rivas F. J., Beltrán F.J., Garcia J.F., Navarrete V. y Gimeno O. (2002). Co-oxidation of p-hydroxybenzoic acid and atrazine by the Fenton's like system $\mathrm{Fe}(\mathrm{III}) / \mathrm{H}_{2} \mathrm{O}_{2}$ J. Hazard. Mater. 91, 143-157. DOI:10.1016/S03043894(01)00381-8.

Safarzadeh A. A., Bolton J. R. y Cater S. R. (1997). Ferrioxalate-mediated photodegradation of organic pollutants in contaminated water. Water Res. 31, 787798. DOI:10.1016/S0043-1354(96)00373-9.

Salem A. M., Abdel-Halim S. T., El-Sawy E. M. A. y Zaki B. A. (2009). Kinetics of degradation of allura red, ponceau $4 \mathrm{R}$ and carmosine dyes with potassium ferrioxalate complex in the presence of $\mathrm{H}_{2} \mathrm{O}_{2}$. Chemosphere 76, 1088-1093. DOI:10.1016/j.chemosphere.2009.04.033.

Saritha P., Aparna C., Himabindu V. y Anjaneyulu Y. (2007). Comparison of various advanced oxidation processes for the degradation of 4-chloro-2 nitrophenol. J. Hazard. Mater. 149, 609-614. DOI:10.1016/j. jhazmat.2007.06.111.

Selvam K., Muruganandham M. y Swaminathan M. (2005). Enhanced heterogeneous ferrioxalate photofenton degradation of reactive orange 4 by solar light. Sol. Energ. Mat. Sol. Cells. 89, 61-74. DOI:10.1016/j. solmat.2005.01.002.

Shanthi, M., Ginish E. y Rajamanickam D. (2013). Photocatalytic degradation of an organic pollutant, benzyl alcohol using an enhanced solar photo Fenton process. International Journal of Chem. Tech. Research 5, 2521-2533. 
Sistema Estadístico de comercio exterior SIEX. Clasificación según Uso o Destino [en linea]. http://websiex. dian.gov.co/pls/siex/impo_cuode\$.startup 03/01/2015.

Tran T., Thuy T., Feng H. y Cai Q. (2013). Photocatalytic degradation of pentachlorophenol on $\mathrm{ZnSe} / \mathrm{TiO}_{2}$ supported by photo-Fenton system. Chem. Eng. J. 223, 379-387. DOI:10.1016/j.cej.2013.03.025.
Vedrenne M., Vasquez M. R., Prato G. D., Frontana U. B. A., Hernandez E. M. y de Andrés J. M. (2012). A ferrous oxalate mediated photo-Fenton system: Toward an increased biodegradability of indigo dyed wastewaters. J. Hazard. Mater. 243, 292-301. DOI:10.1016/j. jhazmat.2012.10.032. 